\title{
Segmental Body Composition Assessment for Obese Japanese Adults by Single-Frequency Bioelectrical Impedance Analysis with 8-point Contact Electrodes
}

\author{
Susumu Sato ${ }^{1)}$, Shinichi Demura ${ }^{2)}$, Tamotsu Kitabayashi ${ }^{3)}$ and Takanori Noguchi ${ }^{4)}$ \\ 1) Life-long sports core, Kanazawa Institute of Technology \\ 2) Natural Science and Technology, Kanazawa University \\ 3) Yonago National College of Technology \\ 4) Kanazawa University Graduate School of Science and Technology
}

\begin{abstract}
This study aimed to determine the accuracy of segmental body composition variables estimated by singlefrequency BIA with 8-point contact electrodes (SF-BIA8), compared with dual-energy X-ray absorptiometry (DXA). Subjects were 72 obese Japanese adults (43 males and 29 females) aged 30 to 66 years. Segmental body composition variables (fat free mass: FFM, fat mass: FM, and percent fat mass: \%FAT) were measured by these techniques. The correlations between impedance values and FFM measured by DXA were calculated. To examine the consistency in predicted values (SF-BIA8) with the reference (DXA), significant mean differences were tested by t-test and the degree of the difference was assessed by effect size. Correlations between the reference and predicted values were calculated. Additionally, the standard error of estimation (SEE) when estimating the reference from the predictor and the relative value of the SEE to the mean value of the DXA measurement (\%SEE) were calculated. Systematic error was examined by Bland-Altman plots. High correlations were found between impedance and FFM measured by SF-BIA8. FFM in the extremities showed high correlations with the reference values, but systematic error was found. SF-BIA8 tended to overestimate FFM in the trunk. The consistencies in \%FAT and FM with the reference value are inferior to those for FFM, and SEE values in \%FAT and FM were greater than those for FFM. The accuracy of the estimated values in the trunk (FFM, $\%$ FAT, and FM) are inferior to those of the total body and extremities. J Physiol Anthropol 26(5): 533-540, 2007 http://www.jstage.jst.go.jp/browse/jpa2

[DOI: 10.2114/jpa2.26.533]
\end{abstract}

Keywords: fat free mass, fat mass, percent fat, OctapolarBIA, DXA

\section{Introduction}

Bioelectrical impedance analysis (BIA) systems that are based on contact electrodes have improved simplicity in measurement as compared to BIA systems based on conventional gel electrodes (Pietrobelli et al., 2004; Organ et al., 1994; Cable et al., 2001; Bracco et al., 1996; Cha et al., 1997). Various BIA devices based on this new system are being increasingly utilized in clinical and research settings (Pietrobelli et al., 2004; Organ et al., 1994; Cable et al., 2001; Bracco et al., 1996; Cha et al., 1997). In addition, Organ et al. (1994) indicated that an important advance in BIA was to have a simple means by which to make segmental measurements. Recently, BIA devices with eight (two on each hand and foot) contact electrodes were developed, and these devices can estimate segmental body composition (Cha et al., 1997; Sakamoto et al., 2000; Bedogni et al., 2002). Compared with expensive imaging techniques, such as computed tomography (CT), magnetic resonance imaging (MRI), and dual energy Xray absorptiometry (DXA), these BIA devices provide a significantly more simple alternative (Cha et al., 1997; Sakamoto et al., 2000; Bedogni et al., 2002). However, there are few reports on the estimation accuracy of these BIA devices (Pietrobelli et al., 2004; Cha et al., 1997; Bedogni et al., 2002).

In body composition assessment using BIA, fat free mass (FFM) is estimated based on the assumption that the bioelectrical impedance (BI) depends on the cross-sectional area and length of the FFM (Kushner, 1992; Baumgartner, Chumlea and Roche, 1990). Also, in segmental body composition assessment this BI-FFM relationship is available, and segmental FFM can be estimated by determining the segmental BI (Organ et al., 1994; Bracco et al., 1996; Lukaski, 2000). 
In total body composition assessment, fat mass (FM) can be calculated by subtracting the FFM from the total body mass. In segmental body composition, however, it is difficult to determine the weight of each segment (each extremity and the trunk). The BIA system, which can measure segmental fat mass and percent fat mass, estimates these segmental body composition variables by using regression formulas, which consist of the segmental BI value and some anthropometric variables. Unlike the case with total body composition assessment, the estimation accuracy of relative and absolute segmental fat mass is independent of the accuracy of the segmental FFM. Therefore, the accuracy should be examined for the relative and absolute fat mass as well as for the FFM in each segment.

Pietrobell et al. (2004) examined the accuracy of this SFBIA8 for 40 subjects (20 males and 20 females) with a wide range of characteristics in age and BMI, but they did not examine the accuracy independently for each of the male and female groups. Since the regression formulas used in the BIA system are developed for each sex group, their accuracy should be examined according to sex group. Furthermore, since the applicable range of the regression formula depends on the sample characteristics, it is important to examine the accuracy of this SF-BIA8 system for the obese population.

This study aimed to examine the accuracy of segmental body composition assessment using the SF-BIA8 system for male and female obese Japanese adults and to clarify the utility and problems of this BIA system.

\section{Methods}

\section{Subjects}

The subjects were 72 obese $\left(\mathrm{BMI} \geq 25 \mathrm{~kg} / \mathrm{m}^{2}\right)$ Japanese adults aged 30 to 66 years, of which 43 were males and 29 were females. The definition of obese was based on the criterion for obesity disease in Japan in relation to obesityrelated complications (Examination Committee of Criteria for 'Obesity Disease' in Japan, Japan Society for the Study of Obesity, 2002). Their mean physical characteristics are shown

Table 1 Characteristics of study sample

\begin{tabular}{lcccc}
\hline & & Males & Females & Total \\
\hline Number & & 43 & 29 & 72 \\
Age & $(\mathrm{yr})$ & $51.0 \pm 9.5$ & $51.4 \pm 9.5$ & $51.2 \pm 9.4$ \\
& & $(30.7-66.1)$ & $(33.6-64.6)$ & $(30.7-66.1)$ \\
Height & $(\mathrm{cm})$ & $168.3 \pm 4.9$ & $153.9 \pm 5.3$ & $162.5 \pm 8.7$ \\
& & $(158.5-177.3)$ & $(142.0-167.5)$ & $(142.0-177.3)$ \\
Body mass $(\mathrm{kg})$ & $79.1 \pm 6.3$ & $66.5 \pm 7.2$ & $74.0 \pm 9.1$ \\
& & $(66.7-90.6)$ & $(51.0-81.5)$ & $(51.0-90.6)$ \\
BMI & $\left(\mathrm{kg} / \mathrm{m}^{2}\right)$ & $27.9 \pm 1.7$ & $28.1 \pm 2.7$ & $28.0 \pm 2.2$ \\
& & $(25.2-32.9)$ & $(25.3-35.4)$ & $(25.2-35.4)$ \\
\hline
\end{tabular}

Results are expressed as Mean \pm SD (range).

Sample sizes of each sex and age group were as follows:

Males $(30 \mathrm{~s}=6,40 \mathrm{~s}=12,50 \mathrm{~s}=13,60 \mathrm{~s}$ and over $=12)$

Females $(30 s=6,40 s=5,50 s=11,60 \mathrm{~s}$ and over $=7)$ in Table 1.

After an explanation of the measurement procedure, they were measured by BIA and DXA methods. Prior to the measurements, all subjects were asked to fast for two hours, to void their bladders, and to avoid exercise. Subjects were allowed to wear only swim suits or a light cotton shirt during measurements. To examine test-retest reliability, 45 subjects underwent two trials for SF-BIA8, both of which were conducted during the same day. After the subjects sat in a resting state for five minutes, two SF-BIA trials were conducted consecutively. The study was approved by the Human Subject Ethical Committee of Kanazawa University, and informed consent was provided by each subject prior to participation in the study.

\section{Measurements of SF-BIA8}

This study used a bioelectrical impedance analyzer (BC118, Tanita Corp., Tokyo, Japan), which is a single frequency BIA system $(50 \mathrm{kHz})$ with 8 -point contact electrodes. This system uses two stainless-steel rectangular foot pad electrodes fastened to a metal platform set on force transducers for weight measurement. There are four separate foot-pad electrodes mounted on the system's base and two electrodes in each of the hand grips (Pietrobelli et al., 2004). This BIA system can estimate body composition in either "standard mode" or "athlete mode." In this study, body composition assessment was conducted in "standard mode." We were careful not to contact the skin of the thigh or axilla during BIA measurement.

This BIA system can evaluate body composition (FFM, FM, percent fat: \%FAT) indirectly at each extremity (right arm, left arm, right leg, left leg) and trunk (trunk and head). The regression formulas used to estimate segmental body composition variables were developed based on DXA measurements.

FFM and \%FAT in each extremity are estimated from a multiple regression formula using the FFM and \%FAT of each segment as measured by DXA as the dependent variable and the height-adjusted impedance for each segment, height, body mass and age as the independent variables. The FM in each extremity was calculated from the segmental FFM and \%FAT using the following equation, where "FFM $(\mathrm{kg}) /(1-\% \mathrm{FAT} / 100)$ " is considered to be equal to the weight of the segment:

$$
\mathrm{FM}(\mathrm{kg})=\mathrm{FFM}(\mathrm{kg}) /(1-\% \mathrm{FAT} / 100)-\mathrm{FFM}(\mathrm{kg})
$$

This equation $(\mathrm{FM}=\mathrm{FFM} /(1-\% \mathrm{FAT} / 100)-\mathrm{FFM})$ is based on a two-component model and calculates FM by subtracting FFM from the "weight of the segment." However, when estimating the segmental body composition in this BIA system, the "weight of the segment (each extremity)" is unknown, and \%FAT and FFM in each extremity are predicted from other multiple regression equations. The "relative value of the FFM (\%FFM)" is, therefore, calculated from "\%FAT" $(1-\% \mathrm{FAT}=\% \mathrm{FFM})$, and the value obtained by dividing FFM 
by "\%FFM" is considered to be the "weight of the segment."

Comparing this equation to the total body composition assessment, FM, \%FAT, FFM, and "weight of the segment" are equal to body fat mass (BF), percent body fat (\%BF), lean body mass (LBM), and body mass, respectively. For example, for an adult with a body mass of $100 \mathrm{~kg}$ and $\% \mathrm{BF}$ of $30 \%, \mathrm{BF}$ and $\mathrm{LBM}$ are $30 \mathrm{~kg}$ and $70 \mathrm{~kg}$, respectively. In this equation, $\mathrm{BF}$ is calculated as follows:

$$
\begin{aligned}
\mathrm{BF} & =\text { body mass }-\mathrm{LBM} \\
& =\mathrm{LBM} / \% \mathrm{LBM}-\mathrm{LBM}=\mathrm{LBM} /(1-\% \mathrm{BF} / 100)-\mathrm{LBM} \\
& =70 /(1-0.3)-70=70 / 0.7-70=100-70=30
\end{aligned}
$$

Thus, the accuracy of the FM in each extremity depends on the accuracy of the \%FAT and FFM in each extremity.

In this BIA system, the trunk measurement is calculated by subtracting the sum of the extremities measurements from the total body measurement. For this reason, the trunk measurement more accurately means "trunk and head." However, there is really very little influence of the head in the estimate of body composition in the total body and each extremity, and the trunk measurement obtained from this BIA system is considered as the measurement of just the "trunk." The FFM in the trunk $\left(\mathrm{FFM}_{\text {trunk }}\right)$ is calculated from FFM total body and the FFM values of the extremities in the following formula:

$$
\begin{aligned}
\mathrm{FFM}_{\text {trunk }}= & \mathrm{FFM}_{\text {total body }}-\left(\mathrm{FFM}_{\text {right arm }}\right. \\
& \left.+\mathrm{FFM}_{\text {left arm }}+\mathrm{FFM}_{\text {right leg }}+\mathrm{FFM}_{\text {left leg }}\right)
\end{aligned}
$$

Similarly, the FMtrunk is calculated by the following formula:

$$
\begin{aligned}
\mathrm{FM}_{\text {trunk }}= & \mathrm{FM}_{\text {total body }} \\
& -\left(\mathrm{FM}_{\text {right arm }}+\mathrm{FM}_{\text {left arm }}+\mathrm{FM}_{\text {right leg }}+\mathrm{FM}_{\text {left leg }}\right)
\end{aligned}
$$

Since the accuracy of FM in the trunk also depends on the accuracy of \%FAT and FFM in each extremity, this is a limitation of the method.

\section{DXA measurement}

A dual-energy X-ray absorptiometry system (DPX-L; Lunar Radiation Corp., Madison, WI; whole body scanning, software version 1.3Z) was used. Bone mineral content (BMC), FM and bone-free lean tissue mass (LTM) were measured from the differences in absorption rates when penetrated by two $\mathrm{x}$-rays, one high-energy $(80-100 \mathrm{keV})$ and the other low-energy $(40-50 \mathrm{keV})$. A trained radiology technician performed the measurements on subjects who were wearing only a light cotton shirt to minimize clothing absorption. DXA measurements were performed following standard procedures according to the manufacturer's guidelines while the subject was lying in a supine position on a table. The whole body scanning time was $20 \mathrm{~min}$, and the total X-ray irradiation absorbed by the patient was 5mrems or lower, which corresponds to $10 \%$ of a standard chest X-ray film.

\section{Statistical analyses}

The test-retest reliability of impedance values of the right arm, left arm, right leg, left leg and whole body (between right arm and right leg) were examined by calculating the intra-class correlation coefficient. The difference between trials was examined by analysis of variance (ANOVA). The consistency and estimation biases were examined for the SF-BIA8 variables of FFM, FM and \%FAT. Mean differences between the two methods (DXA and SF-BIA8) were assessed with the Student's paired t-test, and the degree of the difference was assessed by effect size (ES). Relationships between these methods were determined by Pearson's correlation coefficient.

\begin{tabular}{|c|c|c|c|c|}
\hline & $1 \mathrm{st}$ & 2nd & \multirow{2}{*}{ ANOVA } & \multirow{2}{*}{$\mathrm{ICC}$} \\
\hline & Mean $\pm S D$ & Mean \pm SD & & \\
\hline Right leg & $224.9 \pm 25.1$ & $224.5 \pm 24.8$ & $\mathrm{~ns}$ & 0.99 \\
\hline Left leg & $224.8 \pm 27.4$ & $224.3 \pm 26.6$ & $\mathrm{~ns}$ & 0.99 \\
\hline Right arm & $298.5 \pm 40.0$ & $297.5 \pm 37.9$ & ns & 0.99 \\
\hline Left arm & $304.2 \pm 40.0$ & $305.0 \pm 40.3$ & ns & 0.98 \\
\hline Total body & $552.3 \pm 66.8$ & $550.9 \pm 64.7$ & ns & 0.99 \\
\hline
\end{tabular}
The standard error of estimation (SEE) was calculated by regression analysis when using the DXA measurement as the dependent variable and SF-BIA8 measurement as the independent variable. The relative values of the SEE $(\% \mathrm{SEE})$ for the mean value of the DXA measurements were calculated.

Table 2 Reliability of impedance values in SF-BIA8

Reliability was examined for 45 subjects.

ICC: Intra-class correlation coefficient

ns: not significant

Table 3 Relationships between height-adjusted impedance and FFM measured by DXA

\begin{tabular}{rccccc}
\hline \multicolumn{7}{c}{ Right leg } & Left leg & Right arm & Left arm & Total body \\
\hline \multicolumn{7}{c}{ Mean \pm SD values of impedance $(\Omega)$} \\
Total & $223.5 \pm 24.7$ & $223.3 \pm 25.4$ & $295.5 \pm 39.8$ & $301.1 \pm 41.3$ & $548.1 \pm 64.8$ \\
Males & $215.1 \pm 20.5$ & $214.1 \pm 19.9$ & $273.4 \pm 25.2$ & $277.9 \pm 26.8$ & $514.6 \pm 45.9$ \\
Females & $236.0 \pm 25.3$ & $236.9 \pm 26.8$ & $328.4 \pm 34.4$ & $335.5 \pm 34.7$ & $597.9 \pm 56.7$ \\
Relationship (r) between DXA and height-adjusted impedance & & \\
Total & $0.88^{* *}$ & $0.86^{* *}$ & $0.95^{* *}$ & $0.94^{* *}$ & $0.93^{* *}$ \\
Males & $0.68^{* *}$ & $0.64^{* *}$ & $0.81^{* *}$ & $0.81^{* *}$ & $0.79^{* *}$ \\
Females & $0.81^{* *}$ & $0.65^{* *}$ & $0.88^{* *}$ & $0.83^{* *}$ & $0.77^{* *}$ \\
\hline
\end{tabular}

Height-adjusted impedance: impedance/height $(\mathrm{m})^{2},{ }^{* *}: p<0.01$ 
Systematic error and the limits of agreement between the two methods (defined as the mean $\pm 2 \mathrm{SD}$ of the difference between the methods) were examined by Bland-Altman plots (Bland and Altman, 1986). The estimation errors (DXA minus SF-BIA8) were plotted against the mean of the DXA and SFBIA8 measurements, and the correlation between the errors and the mean of the DXA and SF-BIA8 measurements was calculated. The relative value of the error $(\%$ Error) for the mean value of the DXA and SF-BIA8 measurements was calculated. Significance in this study was set at $p<0.05$.

\section{Results}

\section{Test-retest reliability of impedance values in SF-BIA8}

For the test-retest reliability in impedance values of the SFBIA8, there were no significant differences in values between trials, and intra-class correlation coefficients for impedance values were greater than 0.98 (Table 2). Intra-class correlation coefficients for body composition variables (FFM, FM, and $\% \mathrm{FAT}$ ) were as follows: FFM, 0.99 (both arms and trunk) to 1.0 (both legs and total body); FM, 0.98 (trunk) to 1.0 (left arm); \%FAT, 0.99 (both arm, trunk and total body) to 1.0 (both arms).

\section{Relationships between height-adjusted impedance and FFM measured by DXA}

Table 3 shows descriptive statistics of impedance values measured by SF-BIA8 and relationships between impedance and FFM measured by DXA. The correlations between heightadjusted impedance and FFM ranged from 0.86 to 0.95 in the total sample, from 0.64 to 0.81 in males and from 0.65 to 0.88 in females.

\section{Comparisons between DXA and SF-BLA8 measurements}

Table 4 shows the results of the consistency between DXA and SF-BIA8 measurements. Figure 1 shows a plot of the segmental body composition variables (FFM, \%FAT, and FM) estimated by DXA and SF-BIA8 for each segment (right leg, right arm and trunk) and for the total body. Furthermore, Fig. 2 plots the error (DXA-SF-BIA8) against the mean value of DXA and SF-BIA8 measurements and shows the correlations between them.

\section{1) $F F M$}

Although significant mean differences in FFM values between DXA and SF-BIA8 were found, effect sizes (ES) tended to be low except for those of the trunk. As shown in Fig. 1, FFM values estimated by SF-BIA8 almost agreed with DXA measurements, except for in the trunk. However, systematic error was found in the legs (both sexes) and arms (females) (Fig. 2). SF-BIA8 tended to overestimate FFM in the trunk in both males and females, and their ES values were high $(E S>2.0)$, although there were no systematic errors in FFM in the trunk or total body.
Table 4 Consystemcy of DXA and SF-BIA8 measurements

\begin{tabular}{|c|c|c|c|c|c|}
\hline \multirow{2}{*}{ Variable } & \multirow{2}{*}{ Segment } & \multirow{2}{*}{$\frac{\mathrm{DXA}}{\text { Mean } \pm S D}$} & \multirow{2}{*}{$\frac{\text { SF-BIA8 }}{\text { Mean } \pm \text { SD }}$} & \multirow{2}{*}{ t-value } & \multirow{2}{*}{ ES } \\
\hline & & & & & \\
\hline \multicolumn{6}{|l|}{ Total $(n=72)$} \\
\hline \multirow[t]{6}{*}{ FFM $(\mathrm{kg})$} & Right leg & $8.6 \pm 1.8$ & $8.5 \pm 1.7$ & $1.88 \mathrm{~ns}$ & 0.07 \\
\hline & Left leg & $8.5 \pm 1.8$ & $8.5 \pm 1.7$ & $0.61 \mathrm{~ns}$ & 0.02 \\
\hline & Right arm & $2.7 \pm 0.6$ & $2.7 \pm 0.6$ & $1.29 \mathrm{~ns}$ & 0.04 \\
\hline & Left arm & $2.7 \pm 0.7$ & $2.6 \pm 0.6$ & $3.7^{* *}$ & 0.11 \\
\hline & Trunk & $21.7 \pm 3.8$ & $27.1 \pm 5.1$ & $22.42 * *$ & -1.20 \\
\hline & Total body & $48.8 \pm 9.0$ & $49.6 \pm 9.7$ & $2.13^{*}$ & -0.08 \\
\hline \multirow[t]{6}{*}{$\mathrm{FM}(\mathrm{kg})$} & Right leg & $3.8 \pm 1.0$ & $4.1 \pm 0.8$ & $3.86^{* *}$ & -0.31 \\
\hline & Left leg & $3.7 \pm 1.0$ & $4.1 \pm 0.9$ & $4.37^{* *}$ & -0.35 \\
\hline & Right arm & $1.1 \pm 0.3$ & $1.1 \pm 0.3$ & $2.24 *$ & 0.15 \\
\hline & Left arm & $1.1 \pm 0.3$ & $1.1 \pm 0.3$ & $0.95 \mathrm{~ns}$ & 0.07 \\
\hline & Trunk & $15.2 \pm 2.4$ & $14.2 \pm 2.7$ & $4.06^{* *}$ & 0.36 \\
\hline & Total body & $25.8 \pm 4.4$ & $24.5 \pm 4.8$ & $4.54 * *$ & 0.29 \\
\hline \multirow[t]{6}{*}{$\%$ FAT $(\%)$} & Right leg & $30.9 \pm 7.8$ & $32.9 \pm 7.5$ & $4.08^{* *}$ & -0.26 \\
\hline & Left leg & $30.9 \pm 7.8$ & $32.9 \pm 7.7$ & $3.97^{* *}$ & -0.25 \\
\hline & Right arm & $29.2 \pm 8.8$ & $28.7 \pm 8.2$ & $1.37 \mathrm{~ns}$ & 0.06 \\
\hline & Left arm & $29.3 \pm 8.7$ & $29.3 \pm 9.0$ & $0.1 \mathrm{~ns}$ & 0.00 \\
\hline & Trunk & $41.3 \pm 5.3$ & $34.8 \pm 7.0$ & $13.81^{* *}$ & 1.05 \\
\hline & Total body & $34.9 \pm 6.0$ & $33.6 \pm 7.3$ & $3.53^{* *}$ & 0.20 \\
\hline \multicolumn{6}{|c|}{ Males $(n=43)$} \\
\hline \multirow[t]{6}{*}{ FFM (kg) } & Right leg & $9.8 \pm 1.1$ & $9.7 \pm 0.7$ & $0.84 \mathrm{~ns}$ & 0.09 \\
\hline & Left leg & $9.8 \pm 1.1$ & $9.7 \pm 0.7$ & $0.95 \mathrm{~ns}$ & 0.09 \\
\hline & Right arm & $3.1 \pm 0.4$ & $3.2 \pm 0.3$ & $1.41 \mathrm{~ns}$ & -0.12 \\
\hline & Left arm & $3.2 \pm 0.4$ & $3.1 \pm 0.3$ & $3.38^{* *}$ & 0.27 \\
\hline & Trunk & $24.3 \pm 2.2$ & $30.8 \pm 2.5$ & $25.64^{* *}$ & -2.76 \\
\hline & Total body & $55.2 \pm 4.9$ & $56.7 \pm 4.3$ & $3.23^{* *}$ & -0.34 \\
\hline \multirow[t]{6}{*}{ FM (kg) } & Right leg & $3.4 \pm 0.7$ & $3.7 \pm 0.6$ & $2.67^{*}$ & -0.39 \\
\hline & Left leg & $3.4 \pm 0.7$ & $3.6 \pm 0.6$ & $2.18^{*}$ & -0.31 \\
\hline & Right arm & $0.9 \pm 0.2$ & $0.9 \pm 0.2$ & $0.55 \mathrm{~ns}$ & -0.07 \\
\hline & Left arm & $1.0 \pm 0.2$ & $0.9 \pm 0.1$ & $1.93 \mathrm{~ns}$ & 0.28 \\
\hline & Trunk & $15.2 \pm 2.4$ & $13.3 \pm 2.1$ & $8.8 * *$ & 0.89 \\
\hline & Total body & $24.8 \pm 3.9$ & $22.4 \pm 3.3$ & $7.1^{* *}$ & 0.69 \\
\hline \multirow[t]{6}{*}{$\%$ FAT $(\%)$} & Right leg & $25.8 \pm 4.2$ & $27.3 \pm 2.6$ & $2.39 *$ & -0.42 \\
\hline & Left leg & $25.9 \pm 4.2$ & $27.0 \pm 2.5$ & $1.96 \mathrm{~ns}$ & -0.34 \\
\hline & Right arm & $22.9 \pm 4.2$ & $22.7 \pm 3.1$ & $0.41 \mathrm{~ns}$ & 0.05 \\
\hline & Left arm & $23.1 \pm 4.3$ & $22.6 \pm 2.8$ & $0.94 \mathrm{~ns}$ & 0.13 \\
\hline & Trunk & $38.4 \pm 4.2$ & $30.0 \pm 3.3$ & $16.07^{* *}$ & 2.23 \\
\hline & Total body & $31.0 \pm 3.8$ & $28.3 \pm 2.8$ & $6.03^{* *}$ & 0.80 \\
\hline \multicolumn{6}{|c|}{ Females $(n=29)$} \\
\hline \multirow[t]{6}{*}{$\mathrm{FFM}(\mathrm{kg})$} & Right leg & $6.8 \pm 0.7$ & $6.6 \pm 0.7$ & $2.62 *$ & 0.24 \\
\hline & Left leg & $6.6 \pm 0.7$ & $6.6 \pm 0.7$ & $0.57 \mathrm{~ns}$ & -0.05 \\
\hline & Right arm & $2.1 \pm 0.3$ & $2.0 \pm 0.2$ & $5.84 * *$ & 0.52 \\
\hline & Left arm & $2.0 \pm 0.2$ & $2.0 \pm 0.2$ & $1.64 \mathrm{~ns}$ & 0.14 \\
\hline & Trunk & $17.8 \pm 1.6$ & $21.5 \pm 1.9$ & $14.78^{* *}$ & -2.17 \\
\hline & Total body & $39.3 \pm 3.3$ & $38.9 \pm 3.6$ & $1.34 \mathrm{~ns}$ & 0.12 \\
\hline \multirow[t]{6}{*}{ FM (kg) } & Right leg & $4.3 \pm 1.1$ & $4.7 \pm 0.8$ & $2.78 * *$ & -0.34 \\
\hline & Left leg & $4.2 \pm 1.1$ & $4.7 \pm 0.8$ & $4.27^{* *}$ & -0.51 \\
\hline & Right arm & $1.4 \pm 0.3$ & $1.2 \pm 0.3$ & $4.44 * *$ & 0.42 \\
\hline & Left arm & $1.3 \pm 0.3$ & $1.3 \pm 0.3$ & $0.86 \mathrm{~ns}$ & -0.09 \\
\hline & Trunk & $15.1 \pm 2.5$ & $15.7 \pm 3.0$ & $2.12^{*}$ & -0.22 \\
\hline & Total body & $27.2 \pm 4.9$ & $27.6 \pm 5.1$ & $1.19 \mathrm{~ns}$ & -0.07 \\
\hline$\%$ FAT (\%) & Right leg & $38.5 \pm 5.4$ & $41.3 \pm 3.1$ & $3.5^{* *}$ & -0.63 \\
\hline & Left leg & $38.5 \pm 5.4$ & $41.5 \pm 3.1$ & $3.88 * *$ & -0.70 \\
\hline & Right arm & $38.5 \pm 4.2$ & $37.5 \pm 4.7$ & $1.68 \mathrm{~ns}$ & 0.22 \\
\hline & Left arm & $38.5 \pm 4.2$ & $39.3 \pm 4.8$ & $1.3 \mathrm{~ns}$ & -0.18 \\
\hline & Trunk & $45.6 \pm 3.6$ & $41.9 \pm 4.7$ & $6.39 * *$ & 0.90 \\
\hline & Total body & $40.7 \pm 3.5$ & $41.4 \pm 4.1$ & $1.65 \mathrm{~ns}$ & -0.19 \\
\hline
\end{tabular}

ES: Effect size. ${ }^{* *}: p<0.01, *: p<0.05$, ns: not significant 
FFM(kg)
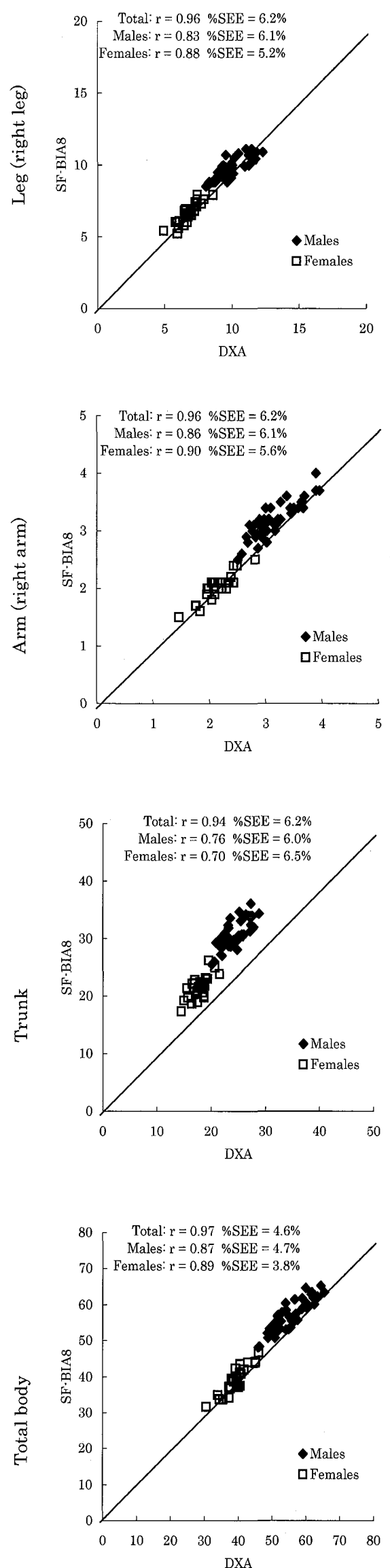

$\% \mathrm{FAT}(\%)$

$\mathrm{FM}(\mathrm{kg})$
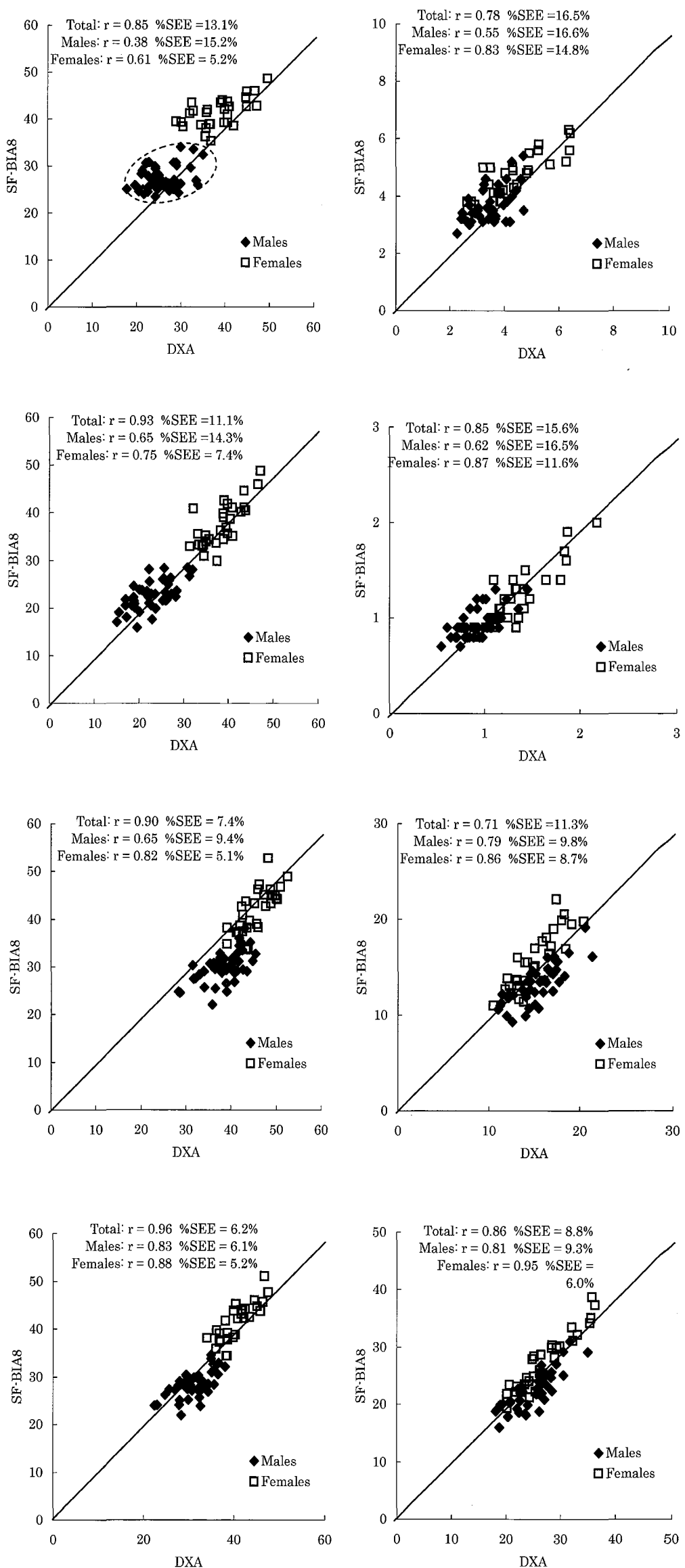

Fig. 1 Consistency between DXA and SF-BIA8 for each segmental body composition variable. r: Correlations between DXA and SF-BIA8 measurements. All correlations were significant $(p<0.05)$. \%SEE: \%SEE is the relative value of the SEE for mean value of DXA measurement. 

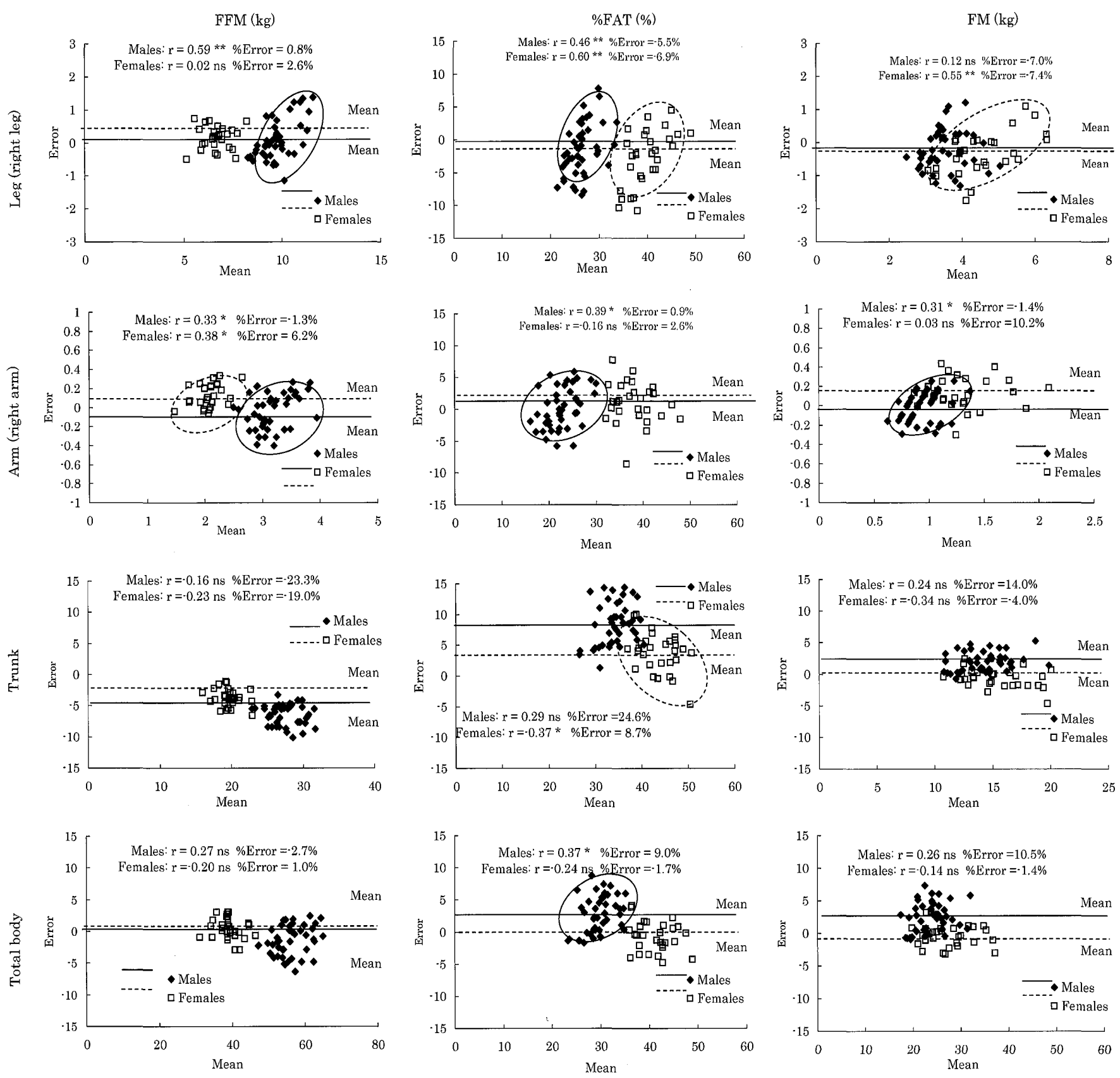

Fig. 2 The error between DXA and SF-BIA8 measurements. Figures plotted error (DXA-SF-BIA8) for mean value of them. Continuous and dashed lines indicate the mean of the error in males and females, respectively. r: Correlations between the error and the mean value. \%Error: Relative value of the error for the mean value. ${ }^{* *}: p<0.01 .^{*}: p<0.05$, ns: not significant.

\section{2) $\% F A T$}

Agreement with DXA measurements in the legs was lower in males (right leg: $\mathrm{r}=0.38, p<0.05$; left leg: $\mathrm{r}=0.40, p<0.01$ ). In the male group, significant moderate correlations $(\mathrm{r}=0.6$ to $0.65, p<0.01$ ) were found in the arms, trunk and total body. In the female group, moderate correlations were found in the legs (right leg: $\mathrm{r}=0.61, p<0.01$; left leg: $\mathrm{r}=0.62, p<0.01$ ), and high correlations over 0.70 were found in other segments and in the total body.

SF-BIA8 tended to underestimate \%FAT in the trunk in both the male and female groups. In the male group, a similar trend was also found in the total body. On the other hand, \%FAT in the legs tended to be overestimated by the SF-BIA8 in both males and females (Figs. 1 and 2). Systematic errors were found in the extremities and in the total body in the male group and in the legs and trunk in the female group (Fig. 2). In both males and females, systematic errors in the legs were greater than for other segments (Fig. 2). The relative values of SEE (\%SEE), which is error when estimating DXA measurements from SF-BIA8 measurements, were greater than those of FFM. This trend was remarkably found in the extremities, and the $\%$ SEE values of \%FAT in the extremities were 2.3 to 2.7 times greater in males and 2.1 to 2.9 times greater in females than those of FFM (Fig. 1). 


\section{3) $F M$}

The trends in consistency with the reference value and systematic error were similar to those of \%FAT. \%SEE values in FM in the extremities tended to be greater than those of FFM. In this study, the \%SEE values in FM were 2.7 to 3.3 times greater in males and 1.2 to 2.3 times greater in females than those of FFM.

\section{Discussion}

This study examined the estimation accuracy of the SFBIA8 (segmental body composition analyzer BC-118, Tanita Corp., which is developed for Japanese subjects) for obese Japanese males and females. Pietrobelli et al. (2004) also examined the estimation accuracy of SF-BIA8 (segmental body composition analyzer, BC-418, Tanita Corp., which is developed for Japanese and Western subjects). They used a study sample with a wide range of characteristics, including age (6 to 64 years) and BMI ( 15.3 to $\left.37.5 \mathrm{~kg} / \mathrm{m}^{2}\right)$, but they did not analyze independently for each of the sex and obesity groups. For both of these SF-BIA8 analyzers, regression formulas for segmental body composition variables were developed for males and for females, and their accuracy should be examined for each respective group. Although the accuracy and applicable range of regression formulas depends on the sample characteristics, the sample characteristics used for the development of these regression formulas were not clearly reported, and the accuracy of this BIA system may vary according to the obesity level of the subjects.

Although this study examined the accuracy of SF-BIA8 for only obese subjects, the results obtained in the total sample support the report by Pietrobelli et al. (2004). Thus, the relationships between height-adjusted impedance and FFM were high $(r>0.86)$, and the values of FFM and \%FAT estimated by SF-BIA corresponded with those measured by DXA (FFM: $r>0.96$; \%FAT: $r>0.85$ ) except for in the trunk. As shown in Fig. 1, however, since the distribution ranges of the segmental and total body composition variables differ according to sex group, there is a possibility that the relationships appear high despite showing a poor relationship when analyzed for each sex group independently. Thus, the accuracy in segmental and total body composition assessments should be examined according to sex group. In this study, there were several problems in estimating segmental body composition by SF-BIA8 for obese Japanese males and females.

In the FFM estimation by SF-BIA8, there were systematic errors in the extremities, although the consistencies with DXA measurements were high both in males and females. Furthermore, FFM in the trunk tended to be overestimated, although there was no systematic error. Estimation of body composition via the bioelectrical impedance technique is based on the relationship between impedance and FFM, in which the impedance depends on the cross-sectional area and length of FFM. For the SF-BIA8 used in this study, the values of FFM in the extremities and in the total body were estimated based on the impedance-FFM relationship. However, since the FFM in the trunk was calculated by the total body value minus the sum of extremities' values, the accuracy of estimating FFM in the trunk is considered to be influenced by the accuracy of the FFM in the extremities and the total body. In contrast, since the relationship between DXA and SF-BIA8 measurements of FFM in the trunk was relatively high $(r>0.70)$ and there were no systematic errors for males or females, accuracy may be improved by correcting the estimation values.

In the \%FAT estimation by SF-BIA8, a low consistency with the DXA measurement in the legs was found in males, and there were systematic errors in the legs in both males and females. Furthermore, the SF-BIA8 tended to overestimate \%FAT in the trunk in both males and females, and systematic error was found in the female group. In general, as in the case of percent total body fat, to estimate relative fat mass, the weight of the segment is required. However, it is difficult to estimate the weight of each leg or arm, unlike the situation in the case of total body mass. Therefore, the SF-BIA8 used in this study independently estimates FFM and \%FAT in the extremities from respective regression formulas. These regression formulas directly estimate DXA measurements (FFM or \%FAT) from height, body mass, age, and heightadjusted impedance. Thus, the differences from the reference values or their systematic errors in \%FAT values in the extremities depend on the accuracy of these regression formulas. In addition, \%FAT in the trunk is calculated by using FFM and FM in the trunk, and FFM and FM in the trunk are estimated from the FFM and FM of the total body and extremities. Thus, all regression formulas used in this SFBIA8 system are involved in \%FAT estimation in the trunk, and the accuracy of \%FAT in the trunk depends on the accuracy of these regression formulas. In this study, estimation error in \%FAT in the trunk were greater compared with other segments, and systematic error was also found in the female group. Although we cannot make further reference to the influence of sex difference on the body fat distribution of the trunk on estimation accuracy, \%FAT in the trunk is considered to be an error-prone variable. Further examination will be required.

In the FM estimation by SF-BIA8, the trends in systematic error and consistency with the reference were similar to those of \%FAT. As shown in \%FAT, \%SEE values in FM in the extremities tended to be greater than those of FFM. These results are considered to be caused by the fact that the FM values in the extremities are calculated based on the \%FAT values in the extremities. Therefore, improvement of the accuracy of the regression formulas of the \%FAT will allow for improvement in the accuracy of the FM values.

The results obtained in this study are thought to be influenced by the characteristics of the subjects as well as the problems inherent in the SF-BIA8 system itself. Although this study used only obese Japanese adults, the influence of sample characteristics on accuracy is not clear, because there is no 
report that has systematically examined the accuracy of SFBIA8 using only non-obese subjects. However, according to the report by Sakamoto et al. (2000), which included a large original group (355 subjects: 188 males, ranging in age from 19 to 72 years and in BMI from 16.2 to $39.2 \mathrm{~kg} / \mathrm{m}^{2}$, and 167 females, ranging in age from 18 to 73 years and in BMI from 16.2 to $41.5 \mathrm{~kg} / \mathrm{m}^{2}$ ) and a cross-validation group (385 subjects: 160 males, ranging in age from 20 to 63 years and in BMI from 16.9 to $36.3 \mathrm{~kg} / \mathrm{m}^{2}$, and 225 females, ranging in age from 18 to 70 years and in BMI from 15.7 to $40.4 \mathrm{~kg} / \mathrm{m}^{2}$ ), the $\%$ FAT in the trunk as estimated by SF-BIA8 was found to be highly correlated to the reference but overestimating the reference. This indicates that the results obtained in this study were not necessarily due only to the influence of using obese subjects.

Although more improvement in prediction equations may be required, there are few devices that can easily and inexpensively estimate segmental body composition in a field setting. The within- and between-day coefficients of variation (CV) in the SF-BIA8 measurements were reported to be small (Pietrobelli et al., 2004). This may suggest that the SF-BIA8 can be used in intrapersonal comparisons, on the understanding the fact that the measurements of SF-BIA8 include errors.

In summary, this study examined the accuracy of the estimation of segmental body composition by the SF-BIA8 system for obese Japanese adults. FFM values in the total body and extremities estimated by SF-BIA8 highly correlated with the reference values, but the systematic error found in the extremities requires attention. The consistencies in \%FAT and FM with the reference values are inferior to those for FFM, and the SEE values in \%FAT and FM were greater than those for FFM. The accuracy of the estimated values in the trunk (FFM, \%FAT, and FM) are inferior to those in the total body and extremities. It is unlikely that the results obtained in this study are due only to the characteristics of the subjects (obese population). This BIA system has a great advantage in that it can easily and inexpensively estimate segmental body composition in a field setting. However, further modification of the prediction equations is required.

\section{References}

Baumgartner RN, Chumlea WC, Roche AF (1990) Bioelectrical impedance for body composition. Exerc Sport Sci Rev 18: 193-224
Bedogni G, Malavolti M, Severi S, Poli M, Mussi C, Fantuzzi AL, Battistini N (2002) Accuracy of an eight-point tactileelectrode impedance method in the assessment of total body water. Eur J Clin Nutr 56: 1143-1148

Bracco D, Thiebaud D, Chiolero RL, Landry M, Burckhardt P, Schutz Y (1996) Segmental body composition assessed by bioelectrical impedance analysis and DEXA in humans. J Appl Physiol 81: 2580-2587

Bland JM, Altman DG (1986) Statistical methods for assessing agreement between two methods of clinical measurement. The Lancet 1: 307-310

Cable A, Nieman DC, Austin M, Hogen E, Utter AC (2001) Validity of leg-to-leg bioelectrical impedance measurement in males. J Sports Med Phys Fitness 41: 411-414

Cha K, Shin S, Shon C, Choi S, Wilmore W (1997) Evaluation of segmental bioelectrical impedance analysis (SBIA) for measuring muscle distribution. J ICHPER SD-ASIA: 11-14

Examination Committee of Criteria for 'Obesity Disease' in Japan, Japan Society for the Study of Obesity (2000) New Criteria for 'Obesity Disease' in Japan. Circ J 66: 987-992

Kushner RF (1992) Bioelectric impedance analysis: A review of principles and applications. J Am Coll Nutr 11: 199-209

Lukaski HC (2000) Assessing regional muscle mass with segmental measurements of bioelectrical impedance in obese women during weight loss. Ann N Y Acad Sci 904: 154-158

Organ LW, Bradham GB, Gore DT, Lozier SL (1994) Segmental bioelectrical impedance analysis: theory and application of a new technique. J Appl Physiol 77: 98-112

Pietrobelli A, Rubiano F, St-Onge MP, Heymsfield SB (2004) New bioimpedance analysis system: improved phenotyping with whole-body analysis. Eur J Clin Nutr 58: 1479-1484

Sakamoto Y, Miura J, Ohomori M, Ikeda Y, Nishizawa M (2000) Clinical availability of segmental bioelectrical impedance analysis in the assessment of segmental body composition. J Occup Health 42: 252 [In Japanese]

Received: October 2, 2006

Accepted: June 6, 2007

Correspondence to: Susumu Sato, Life-long Sports Core, Kanazawa Institute of Technology, Ohgigaoka 7-1, Nonoichi, Ishikawa 921-8501, Japan

Phone: +81-76-248-1100 (ext. 2386)

Fax: +81-76-294-6704

e-mail: sssato@neptune.kanazawa-it.ac.jp 